\title{
IMPROVING UTILIZATION OF CONCEPTUAL AND THEORETICAL FRAMEWORK IN NURSING RESEARCH
}

IDONGESIT I. AKPABIO

\author{
(Received 14 October 2014; Revision Accepted 18 March 2015)
}

\begin{abstract}
The Nursing and Midwifery Councils of many nations including Nigeria and nursing departments of various Universities and institutions have made it mandatory that conceptual and theoretical framework are used to guide Nursing research presented as part of the requirements for the award of certificates and degrees. However, it is often observed that many researchers are not skilled in the use of conceptual and theoretical framework and many do not also utilize conceptual or theoretical framework to guide studies conducted after graduation from their various educational institutions. Others do so for some parts of the research and do not also explain how the framework can be used to guide implementation of the study findings. Additionally, many publishers of research reports do not also demand for inclusion of framework in papers accepted for publication.
\end{abstract}

This paper is aimed at highlighting the importance of using a conceptual framework to guide a study, the criteria that should guide the appraisal and selection of a framework; challenges that can hinder utilization of framework to guide research work and measures of improving utilization of conceptual framework in nursing research.

KEYWORDS: Conceptual, Framework, Nursing, Research, Theoretical.

\section{INTRODUCTION}

In nursing as in many other fields of study, it is required that researchers identify conceptual frameworks, define the concepts in the framework and apply them appropriately based on their relevance to the topics of the research. Utilization of conceptual or theoretical framework could involve the use of already existing theories or conceptual models as basis or guide for the study to generate predictions that could be tested empirically (Akpabio and Ebong, 2010). Sometimes, advanced researchers can construct their models using existing theories or they can modify existing models for use in their research work.

The advantages of using such theoretical or conceptual framework are that the research findings would have broad significance and utility as well as relevant assumptions to direct the study (Kaisser, 2009). To achieve aforementioned benefits, great care should be taken to ensure that the model in use is appropriate and relevant to study the associations between variables and to generate required data for valid results. However, it is often observed that many researchers do not often utilize conceptual/theoretical framework to guide their studies and publishers of research studies do not also often demand for inclusion of framework in papers accepted for publication. Whenever conceptual or theoretical framework is applied to guide a study, the assumptions are usually specified, thus making practical application of the study findings easier.

Various schools of thought exist on whether identifying and defining the theoretical or conceptual frameworks should be done before or after literature review. While one school of thought submits that it should follow literature review since the literature should help to identify the framework needed, another school of thought argues that it should be done prior to literature review so as to guide in formulating study objectives based on the frameworks' variables and in selecting the sub-headings of literature needed (Akpabio and Ebong, 2010; Vaughan, 2008). Whichever choice the researcher makes, conceptual framework should be relevant to guide the study appropriately and provide valid results. Although conceptual framework can be applied to both quantitative and qualitative studies, their use in qualitative studies can be said to be loosely applied compared to the more serious application that may apply in quantitative studies, which is the focus of this paper. This paper is aimed at providing a brief highlight and the importance of conceptual framework, measures of improving utilization of conceptual or theoretical framework to guide nursing research and subsequently the implementation of research findings, the challenges that could be encountered when applying conceptual framework in nursing research and helpful suggestions on how to overcome the challenges.

\subsection{Highlight and importance of Conceptual Framework}

The word 'concept' connotes an image or idea held in the mind. Conceptual framework according to Smyth (2004) is a group of broad ideas and principles taken from relevant fields of enquiry for use in structuring subsequent presentation. Thus conceptual and theoretical framework are broadly defined and organized to provide a rationale or structure for the 
interpretation of information in the study.

According to Polit and Hungler (2014), conceptual frameworks or models represent less formal means of organizing phenomena than theories. While theories represent strict abstract generalizations involving formal and systematic explanations about how phenomena are inter-related, conceptual models or frameworks are less formal and are assembled by virtue of their relevance to a common theme. Thus, theories unlike conceptual frameworks use deductive reasoning to generate ideas that are highly structured while the conceptual model broadly presents an understanding of the phenomenon of interest and therefore more loosely structured, reflecting the assumptions and philosophical views of the model's designer. Conceptual models generate hypotheses to be tested. On their similarities, both theories and conceptual frameworks use concepts (ideas) as building blocks and are often used interchangeably as would be applicable in this paper.

A framework when used should be the conceptual underpinning of a study. To some extent, everyone has a conceptual model because every individual has assumptions and beliefs about events. Everyone has a unique set of concepts guiding the categorization of ideas and information with their belief system. In nursing practice, people view situations differently, and have personalized ways of responding to such situations. This explains the various or diverse theories or models of different nurse scientists. Whether implicit or explicit, a person's conceptual model plays a major role in determining behaviour and practice. Conceptual models therefore guide actions and many nurse scientists organized nursing practice in accordance with their models.

These explanations are provided to emphasize the important place of conceptual framework in guiding nursing research and practice. In recent years, it is observed that nursing research has received a major impetus from educational institutions to be guided by conceptual and theoretical frameworks as a means of providing focus to the study. The aim is to guide the selection of variables that should be included and those that can be logically omitted. Thus, in research, conceptual models provide the essential conceptual, instrumental and methodological focus for guiding the work. The models help to define the concepts from which specific variables are derived; it defines research questions and therefore serve as an overall map for the study.

The use of a specific model will therefore determine to a very great extent the kind of information that could be gathered and the way the information should be organized and interpreted (Fawcett, 2012). According to the author, one specific research may focus on one or few aspects of the variables identified from one or more of the selected conceptual model(s). Furthermore, to verify the effectiveness of the research process, concepts in a conceptual framework should be clearly defined and carefully developed to allow for accurate measurement of identified variables and interpretation of research findings.

Commenting further on the usefulness of theoretical or conceptual framework in research, Hanson (2011) stated that they are skeletons on which to build nursing research. It is from the framework that principles, assumptions and ideas are taken to frame the study. George (2009) similarly explained that the use of conceptual framework in research allows researchers to build upon one another's work thereby building a body of knowledge. Furthermore, she asserted that pragmatic, conceptually-based research helps in accumulating deeper understanding overtime and this moves a discipline such as nursing forward.

Additionally, Kaiser (2009) highlighted the purpose of theories in research to include:

- Identification of meaningful and relevant areas for study

- To propose plausible approaches to health problem

- To develop or refine the study

- To define the concepts and propose relationships between concepts

- To interpret research findings

- To generate nursing diagnosis and guide implementation of findings.

Thus, from the contributions of the various authors, it could be observed that the usefulness of theoretical and conceptual framework in research should cover the length and breadth of the research from the beginning of refinement of the research problem to the end where suggestions and or recommendations are provided.

\subsection{Identification, selection and application of} conceptual Framework in research.

Nurse researchers could use either nursing and non-nursing frameworks or theories to provide a conceptual context for their studies. Although each model focuses on the concepts about persons, environment, health/illness and nursing, each model however defines these concepts differently, links them in diverse ways, and gives different emphasis to the relationships among variables. Moreover, different models emphasize different processes as being central to nursing (Polit and Hungler 2014).

Akpabio and Ebong (2010) stated that conceptual or theoretical frameworks should be carefully identified and selected to guide a study and the criteria that should guide the appraisal and selection of a framework should include comprehensiveness of the contexts; logical congruence; conceptual clarity; level of abstraction; clinical utility and perspective on the issues of interest. Thus, before selecting theoretical or conceptual models, there is need to evaluate the different theories or frameworks available within the topical area of interest. According to Fawcett (2012), comprehensiveness of the contents involves the depth and breadth of the contents. The depth explains the conceptual model that provides adequate description of its constructs, linking the relational propositions of its constructs to one another while the breadth of the contents focus on the broadness of its scope with the ability to provide guidance in various clinical situations and serve as a basis for research.

Logical congruence involves critical reasoning based on judgments driven by nursing knowledge. According to Fawcett (2012), Logical reasoning should highlight strengths and problems inherent in them. The 
criterion of conceptual clarity emphasizes the need for clear identification of the relationship among concepts while abstractions refer to the extent by which concepts are represented in a conceptual model. Sidani (2002) explained that clinical utility refers to the applicability and relevance of the model to the study or practice. This is very important since the definition of concepts in the framework should be able to assist the researcher in understanding assumptions and the ability to predict the interrelationship among variables in the study

Following the selection of a model or models, clear definitions should be given on how the model(s) view person, environment, health, illness and nursing (Basavanthappa, 2009). The concepts contained in the models should be clearly identified, defined and the relationships among the variables clearly stated to bring out their meanings. The relationship among the variables in the conceptual models should then be used as a guide to identify and relate variables in the study.

Similarly, the assumptions and beliefs reflected in the models should be identified and clearly stated and also used to guide the development of assumptions in the study. Additionally, the researcher should identify and clearly state how the concepts in the models, the assumptions and beliefs are applicable to the study through explanation on the relationships among variables in the model(s) as well as those in the research.

Commenting in line with Sidani's (2002) assertion, Kaiser (2009) outlined some measures that should be considered when applying conceptual framework in research to include the following:

i. The problem being investigated should fit into the chosen framework to guide and enrich the values of its findings.

ii. The conceptual definitions should be drawn from the framework.

iii. The data collection instrument should be congruent with the framework.

iv. The findings should be interpreted in light of explanations provided by the framework.

v. Implications derived from the study should be based on the explanatory power of the framework.

Furthermore, Polit and Hungler (2014) gave few descriptions types for theories or conceptual framework with corresponding research designs by stating that for descriptive or exploratory research design, a theory or model that is descriptive should be chosen, for correlation study, explanatory theory or model should be adopted while for experimental, intervention or quasiexperimental studies, predictive theories or models should be chosen.

Emphasizing the importance of appropriate application of conceptual framework in research, Stenber (2015) provided more systematic and clearly defined steps in the application of conceptual framework in research. In her assertion, the steps should include:

- Doing a search of the literature for theories and examination of the various theories to determine what might be appropriate to investigate the research problem.

- Selecting the theory that will guide and direct the research process.
- Conducting a comprehensive review of literature on studies that used the theory where possible.

- Formulating the hypotheses or research questions to be tested using the theory or propositions from within the theory.

- Focusing on the study variables by selecting the instruments that will be used to test the concepts on the basis of the framework.

- Explaining the findings of the study using the theory.

- Drawing conclusions from the results based on the theory.

- Taking a decision on whether the theory is supported by the results of the study.

- Identifying the implications from the results that can be related to the theory.

- Making recommendations for future research to include use of the theory.

It is important to state that one of the problems on implementation of research findings persist due to the fact that the researchers do not properly explain the interrelationships between the concepts of the model and the variables used in the study especially when making recommendations following research. Flat level recommendations are made and in real life situations, implementation of all the recommendations at a particular point in time can be difficult if not impossible.

For instance, in Lewin's Force Field Analysis of Change Management (Lewin, 1962), there is emphasis on the need to determine which driving forces could be increased or which restraining forces could be reduced with the least efforts. It could sometimes be easier to reduce the impact of restraining forces than to strengthen driving forces or vice versa. Lewin emphasized that focus and priority on change should be on those factors, which could be achieved with the least efforts before others that could pose problems with implementation. Thus, where the conceptual framework that guided the study is properly utilized to the levels of recommendations and actual guide for implementation of research findings, it can improve the possibility of implementing desirable changes.

\subsection{Challenges associated with and measures of improving utilization of conceptual framework in nursing research}

According to Akpabio and Ebong (2010), the challenges often include:

\subsubsection{Limited knowledge of conceptual models or theories;}

Many researchers lack adequate knowledge regarding existing theories or models and how to apply them to nursing research as well as skills in formulating personal and relevant framework to guide research work whenever the need arises. Those who attempt to utilize conceptual models or theoretical framework adopt those that have the concepts at variance with that required to guide the particular study. It should be stated that learning, understanding and application of theories to actual nursing situations either in research work or in the practical situations could best be achieved and is often carried out at a higher level of education. According to 
Kaiser (2009), research that is done in isolation of the relevant theory results in discreet information or data, which does not add to the accumulated knowledge of the discipline. In his assertion, theory guides the research process, forms the research questions, aids in design selection, analysis and interpretation of findings. It enables the scientists to weave the facts together such that the interpreted relationship would therefore be direct and positive.

\subsubsection{Limited scope of literature review:}

Whenever a nurse has limited knowledge of available models or theories to guide research work, extensive review of literature on theories or models that are relevant to health and nursing as well as those related to the specific area of research could assist to some extent in the ability to identify appropriate framework, define the concepts and predict associations among variables in the study of interest.

\subsubsection{Limited knowledge in understanding concepts within the models.}

\subsubsection{Lack of skills in explaining relationship among variables based on the model's framework: \\ In many instances, it is impossible for one} model or theoretical framework to offer complete concepts that the researcher could use to predict relationships among variables in a particular study. This is so even when the researcher is formulating personal framework to guide a study. To expand the scope of predicting relationships among variables in studies, advanced researchers usually combine two or more conceptual models or theories to guide a research, paying particular attention to remove some of the concepts that are not relevant to explain relationships in the particular study of interest. The requirement is therefore that the researcher should be very skilled in identifying, selecting and applying needed concepts to avoid limiting the scope of investigation, or making the study clumsy and meaningless through unnecessary inclusion of concepts that are not relevant for the particular study.

\subsubsection{Inability to identify and understand assumptions within the model and to relate them to the research variables:}

It is important to state that no researcher would expect that all data could be totally analyzed using all the concepts and assumptions within the selected framework without the risk of limiting the results of the investigation or facing confusion in explanation of associations among the various variables. For this reason, it is necessary to utilize conceptual framework with caution, based on good knowledge and clear understanding of concepts and assumptions within the models. Burns and Grove (2012) noted that conceptual framework is a construction of knowledge bounded by experiences of the person who developed it. Individual's experiences differ and may therefore influence personal ideas and assumptions.
1.4.6. Lack or inadequate skills in appraising the conceptual framework for strengths and weaknesses:

Application of conceptual or theoretical framework does not imply the use of any known framework. Apart from ensuring that the framework is appropriately fit for the problem under investigation in terms of the interrelationships of the concepts and the study variables, the strengths and weaknesses or limitations of the framework must be known and addressed accordingly where possible. The areas to be examined for clear understanding and identification of strengths should include the following:

i. Interrelationships of Concepts:

The four phases or different components should be examined and how they interrelate should be understood: Man, Environment, Health and Nursing. Secondly the theory or model's assumptions about man, environment, health and nursing should be understood in relation to the research problem

ii. Applicability of the concepts to research problem and variables:

For this, it should be possible to formulate assumptions from the research problems based on concepts originating from the theory or model.

iii. Theories must be logical in nature:

It must provide logical and systematic way of nursing situations. The key concepts should apply logically and systematically with the study problems.

iv. Ability to be used for clear explanations:

The theory should be simple in regard to the natural progression of the explanations of concepts between the theory and the variables in the research problem.

v. Where it is applicable, it should be the basis for hypothesis that could be tested.

vi. It should contribute or assist in increasing the body of knowledge within the discipline through the implemented research.

vii. It should be appropriate to guide and improve nursing practice that should arise from the research findings.

viii. It should be consistent with other validated theories, laws and principles but could leave open unanswered questions that need to be investigated.

Furthermore, the theory or model's limitations should be identified and addressed. Based on the limitations and whenever the observations from aforementioned assessment areas point toward negative directions, the researcher could, where possible attempt modification of the weak areas if the theory or model has the potentials of contributing to credibility of the study.

\subsubsection{Lack of interest in applying a model to guide a study:}

Many researchers do not have the interest of utilizing frameworks to guide their studies. However, 
since utilization of conceptual/theoretical framework could improve understanding and implementation of research findings, it is necessary to increase efforts at reducing or eliminating the challenges to encourage utilization of conceptual or theoretical framework in research work.

\section{$1.5 \quad$ Overcoming the challenges}

Conceptual and theoretical frameworks are abstractions that could be difficult to understand. Individuals should therefore increase efforts towards the study of concepts and assumptions of the various existing models and theories. By so doing, they could improve their knowledge of existing models and theories and also develop their skills for modifying or developing conceptual framework for application in research work. This entails systematic studies through reading wide array of literature on the subject matter. Similarly, there is need for researchers to strengthen interest towards the application of conceptual models or theoretical framework to guide studies. Interest could motivate the desire for a closer study of conceptual models and subsequently improve skills necessary in applying models to guide studies.

Additionally, there is need for publishers of research reports to encourage potential authors to include and improve actual explanations of conceptual or theoretical frameworks in research reports submitted for publications. By so doing, greater understanding could be achieved on the interrelationship among variables and assumptions used in the study, thus making implementation of research findings easier. In this regard also, the authors of research reports would develop interest in applying models to guide every aspect of studies including how the models could enhance implementation of the study findings.

\section{CONCLUSION}

Theoretical and conceptual frameworks provide the organization for a study. When no existing theory fits the concepts that the researcher wishes to study, the researcher may construct a conceptual framework that can be applied to the study. Once a theoretical or conceptual framework is selected, it should be used throughout the study. This means that it should guide the entire research process.

\section{REFERENCES}

Akpabio, I. I and Ebong, F. S., 2010. Research methodology and statistics in health and behavioural sciences, Calabar: Eclat $\mathrm{Biz}$ Services.

Basavanthappa, B. T., 2009. Nursing theories, New Delhi: Yaypee Brothers Medical Publishers $(P)$ Ltd.

Becker, M., 1978. The Health belief model and sick role behaviuor, Nursing Digest, 6, 35- 4, In M. FrankStromberg \& S. J. Olsen (Eds.). Instrument for clinical healthcare research. Retrieved on 13 February, 2015 from tcn.sagepub.com/content/12/4/284.refs
Burns, N and Grove, S. K., 2012. The practice of nursing research, $\left(4^{\text {th }}\right.$ ed.), Philadelphia: W. B. Saunder, Retrieved on 12 November, 2014 from http://www.currentnursing.com/nursing_theory/

Fawcett, J. A., 2012. Analysis and evaluation of conceptual models of nursing, Retrieved on 12 November, 2014 from http://libguides.emu.edu/nursing.

George, B. J., 2009. Nursing theories, the base for professional nursing practice, $\left(3^{\text {rd }}\right.$ ed.), Norwalk: Appleton and Lange

Hanson, J., 2011. How to write a conceptual framework for nursing research, Retrieved on 12 November, 2014 from www.ehow.com/how_7488679write-conceptual.

Kaiser, P., 2009. Nursing Research and Theories, Retrieved on 12 November, 2014 from http://www.currentnursing.com/nursingtheory/r.

Lewin, K., 1962. Lewin Force Field Analysis Explained, Updated 2012, Retrieved on 12 November 2014 from www.change-managementcoach.com/force...

Polit, D. E and Hungler, B. P., 2014. Doing your research project: $A$ guide for the first time researchers. Retrieved on 12 February 2015 from

http://books.google.com.ng/books?isbn=033526 4476

Sidani, S., 2002. Intervention theory: Validity and clinical utility, Ontario: InA. Brathwaite (Ed.). Framework for guiding research interventions. Retrieved on $12 \quad$ November 2014 from https://ispub.com/lJANP/6/1/8576

Smyth, R., 2004. Exploring the usefulness of a conceptual framework as a research tool: A researcher's reflections, Issues in Educational Research, 14 (2): 167-180, Retrieved on 12 November 2014 from, http://www.iier.org.au/iier14/smyth.html

Stember, M., 2015. Theoretical framework. A little bit about frameworks. Retrieved on 13 February 2014 from http://users.Ipfw.edu/Stember/339/framework.ht $\mathrm{ml}$

Weller, B. F., 1996. Bailliere's Nurses' Dictionary $\left(22^{\text {nd }}\right.$ ed.). London: Educational Low-Priced Books Scheme (ELBS) with Bailliere Tindall. 
